\title{
PREVALÊNCIA DE DEPRESSÃO NOS IDOSOS ATENDIDOS EM UMA UNIDADE DE SAÚDE PERTENCENTE À ESTRATÉGIA DE SAÚDE DA FAMÍLIA EM NOVA LIMA/MG
}

\author{
Therezinha de Jesus REBELO ${ }^{1}$ \\ Regina Coeli Cançado Peixoto PIRES ${ }^{2}$ \\ Lilian de Almeida CARVALHO ${ }^{3}$
}

1 Médica Pediatra, Mestre em Ciências da Saúde.

2 Cirurgiã-dentista, Doutora em Epidemiologia (UFMG), Mestre em Clínicas Odontológicas (UFMG),

Professora da Faculdade de Odontologia da Universidade de Itaúna-MG.

3 Nutricionista Clínica, Mestre em Ciências da Saúde . RESUMO

Recebido em: 24/05/2013 - Aprovado em: 30/07/2013 - Disponibilizado em: 15/08/2013

O processo de envelhecimento populacional em curso no Brasil vem sido acompanhada pelo aumento da frequência de doenças psiquiátricas na terceira idade, sendo a depressão a desordem mais comum. Em pacientes idosos, a depressão costuma apresentar sintomatologia atípica, o que muitas vezes dificulta a sua identificação na prática clínica. Neste trabalho foi determinada a prevalência da depressão em pacientes com idade igual ou superior a 60 anos pertencentes ao Programa de Saúde da Família do Município de Nova Lima/MG, atendidos na Unidade de Saúde Mingú. Participaram deste estudo 97 pacientes, onde 72 (74,2\%) eram do sexo feminino e 25 $(25,8 \%)$ do sexo masculino, sendo a idade média de 70,69 anos. Os possíveis casos de depressão foram identificados com o auxílio da Escala de Depressão Geriátrica em versão reduzida Yesavage (GDS-15), amplamente utilizada e validada como instrumento para diagnóstico de depressão em pacientes idosos. Após a pontuação final, verificou-se que $36(37,2 \%)$ idosos apresentam depressão variando de leve a grave, tendo chamado a atenção o fato de que todos os casos graves eram do sexo feminino. Como a depressão tem implicações negativas importantes na vida do idoso, ficou evidente a necessidade de dirigir especial atenção à esta população que tem aumentado significativamente, para que os possíveis casos sejam identificados e para que intervenções mais eficazes sejam direcionadas a melhoria da qualidade de vida destas pessoas.

Palavras Chaves: Depressão, Qualidade de Vida, Idosos, Epidemiologia, Yesavage.

\section{ABSTRACT}

The population aging process underway in Brazil has been accompanied by increased frequency of psychiatric disorders in the elderly, being depression the most common disorder. In elderly patients, depression often presents atypical symptoms, which often hinders their identification in clinical practice. This study addresses the prevalence of depression in patients aged over 60 belonging to the Family Health Program of the municipality of Nova Lima / MG, treated in the Unity Health Mingú. The study included 97 patients, 72 (74.2\%) were female and $25(25.8 \%)$ males, mean age of 70.69 years. Possible cases of depression were identified using the Geriatric Depression Scale in a shorter version Yesavage (GDS-15), widely used and validated as an instrument for diagnosing depression in elderly patients. After the final score, it was found that 36 (37.2\%) elderly people have depression ranging from mild to severe and draws attention the fact that all severe cases were female. Because depression has significant negative implications on the elderly's life, it was evident the needs to direct special attention to this population that has increased significantly, so that possible cases are identified and more effective interventions are directed at improving quality of life of these people.

Key words: Depression, quality of life, elderly, epidemiology, Yesavage. 


\section{INTRODUÇÃO}

O aumento da expectativa de vida e o envelhecimento da população mundial tem sido motivo de pesquisas em várias áreas da ciência. A Pesquisa Nacional por Amostra de Domicílios (PNAD) realizada em 2004 mostra que a população de idosos já ultrapassa os 17 milhões de indivíduos, correspondendo a cerca de $10 \%$ da população brasileira1. De acordo com o Instituto Brasileiro de Geografia e Estatística (IBGE), a vida média da população no Brasil atingirá, em 2050, o patamar de 81,3 anos. A Organização Mundial da Saúde (OMS) prevê que em 2025 a população com 80 anos ou mais será o grupo etário de maior crescimento, dando ao Brasil a sexta posição entre os países com os mais elevados índices de idosos do mundo (SOUZA, 2003). Neste cenário, fica claro que o aumento da longevidade revela-se como um desafio às sociedades, aos pesquisadores e aos gestores de saúde, devido a importância de se agregar qualidade e significado a esses anos adicionais de vida.

As causas do envelhecimento populacional estão relativamente bem definidas, destacando-se a diminuição dos coeficientes de mortalidade e das taxas de fecundidade e natalidade (SILVA, 2005). Entretanto, os fatores que afetam negativamente a qualidade de vida dos indivíduos na fase designada terceira idade são bastante complexos. Mudanças que podem levar ao questionamento dos valores existenciais, como a perda do cônjuge e as limitações e/ou restrições em se realizar atividades, são frequentemente experimentados pelo idoso. Além disso, a transição demográfica e epidemiológica brasileira, ao contrário do ocorrido em países desenvolvidos, não é acompanhada de melhorias nas condições sociais, econômicas e de assistência à saúde desta população (RAMOS, 1997). Tais aspectos, dentre muitos outros, podem potencialmente comprometer a qualidade de vida destas pessoas (VASCONCELOS, 2007). Neste cenário, pode ter início uma desestruturação psíquica do indivíduo, sendo a depressão a síndrome psiquiátrica mais prevalente nessa faixa etária (CORRÊA, 1997; SHMUELY, 2001).

Reconhecidamente de natureza multifatorial, a depressão está associada a diversos aspectos de ordem biológica, psicológica e social (TEIXEIRA, 2007; GUIMARÃES, 2006). Entre as principais sintomatologias depressivas, destacam-se o humor deprimido, tristeza, desinteresse, desânimo e ideação suicida (IRIGARY e SCHNEIDER, 2007; FREITAS, 2002; MORAES, 2007). Em pacientes idosos, além dos sintomas mais comumente encontrados, podem surgir adicionalmente queixas somáticas, dores crônicas, distúrbios do apetite, irritabilidade, sentimento de inutilidade, fadiga fácil, inquietação e impaciência (FERRARI e DELACORTE, 2007; PAYKEL e PRIEST, 1992; STELLA, 2002). Essa condição normalmente está 
relacionada a fatores como perdas, doenças, carências, aspectos sociais, abandono, dificuldade em se adaptar e à proximidade da morte (AVILA e BOTTINO, 2006; ESTELLA, 2002).

Embora a depressão seja um problema de saúde frequente entre os idosos, a sua identificação é de difícil detecção na prática clínica, sendo ainda pouco diagnosticada e tratada (LEITE, 2006; FLECK et al., 2002). Nos idosos, o diagnóstico é dificultado devido a problemas físicos coexistentes, pela predominância dos fatores atípicos da depressão e pelos sintomas depressivos serem erroneamente considerados como uma manifestação comum do processo de envelhecimento (SNOWDON, 2002). As principais consequências da depressão são perda da autonomia, agravamento de quadros patológicos preexistentes, aumento da utilização dos serviços de saúde, adesão reduzida aos tratamentos terapêuticos, aumento do risco de suicídio, redução da qualidade de vida, aumento da morbidade e mortalidade (DJERNES, 2006; PARADELA et al., ALMEIDA, 2002). Em virtude desses fatos, torna-se importante o conhecimento dessa condição e dos fatores a ela associados, sendo este, um passo essencial no processo de planejamento das ações de saúde.

O idoso deprimido normalmente apresenta piora do seu estado geral, o que evidencia a importância do diagnóstico e do tratamento desse distúrbio. Entretanto, a definição de depressão não é consensual, existindo diferentes classificações para a doença. As mais utilizadas são a Classificação Internacional de Doenças (CID-10) e aquela incluída no Manual de Diagnóstico e Estatística das Perturbações Mentais (Diagnostic and Statistical Manual of Mental Disorders - DSM-IV) (MARQUES, 2007). Contudo, devido à complexidade e dificuldade de aplicação de ambas, tornou-se necessário que outros instrumentos, como as escalas psicométricas, fossem utilizados na identificação dos doentes deprimidos.

Em 2009, Aros e Yoshida realizaram um estudo onde os autores fizeram uma busca por instrumentos empregados em pesquisas de avaliação da depressão, onde foi verificado que a aquele do tipo autorrelato é o mais frequente, possivelmente por serem aplicados de forma mais rápida e com menores custos. Em relação às pesquisas brasileiras, seguindo a tendência mundial, a Escala de Depressão Geriátrica (Geriatric Depression Scale - GDS) apresenta-se como um dos instrumentos de avaliação mais utilizados para a identificação de sintomas depressivos em idosos. A GDS foi elaborada especificamente para essa população, considerando a sintomatologia atípica que acomete esses indivíduos, quando comparada a outras faixas etárias (MONTORIO, 1996; YESAVAGEA, 1983). Existem duas versões, sendo uma longa com 30 perguntas (GDS-30) e outra, curta, com 15 (GDS-15), (SOUZA, 2007). Neste 
questionário não são incluídas queixas de desordens somáticas e as respostas são objetivas e dicotômicas em formato simples, sendo propositalmente escolhidas para melhor aceitação do paciente idoso (YESAVAGEA, 1983).

Em 2006, Oliveira et al. aplicaram a escala GDS-15 em idosos participantes de um centro de convivência, onde se verificou que $26 \%$ dos entrevistados apresentavam depressão leve a moderada e $4 \%$ apresentavam depressão grave (OLIVEIRA et al., 2006), Observou-se, ainda, que quanto maior a idade, maior a tendência à depressão. Ferrari e Dalacorte 2007, utilizando a também a GDS15, avaliaram a tendência à depressão em 50 idosos hospitalizados, tendo sido verificado um índice de $38 \%$ para a existência de depressão leve a moderada, além de $8 \%$ apresentarem depressão grave.

Neste trabalho a Escala de Depressão Geriátrica de Yesavage em versão reduzida (EDG-15) é utilizada para se verificar a prevalência da manifestação depressiva em pacientes com idade igual ou superior a 60 anos, pertencentes à Estratégia de Saúde da Família do Município de Nova Lima - MG, atendidos na Unidade de Saúde Mingú.

\section{MÉTODO}

Este é um estudo transversal e descritivo realizado na Unidade de Saúde Mingú, que pertence á Estratégia de Saúde da Família de Nova Lima/ MG, conveniado aos cursos da área de saúde da Universidade Vale do Rio Doce - UNINCOR, campus Mário Pena. Foram entrevistados 97 pacientes de ambos os sexos, sendo considerados elegíveis para o estudo aqueles com idade igual ou superior a 60 anos regularmente cadastrados na Estratégia de Saúde da Família de Nova Lima/MG, atendidos nesta Unidade de Saúde, e que consentiram em participar do estudo formalmente, configurado após a assinatura do Termo de Consentimento Livre e Esclarecido.

Foram considerados não elegíveis para o estudo os portadores de diagnóstico prévio de quadro depressivo com início antes dos 50 anos de idade e/ou história de uso de antidepressivos antes dos 40 anos de idade; os suspeitos e/ou com confirmação diagnóstica de quadro demencial ou intelectualmente incapacitante, independente do motivo; pacientes que solicitassem a exclusão formal do trabalho, até a fase de coleta de dados; pacientes que apresentassem alteração na cognição determinada por demência préexistente e ausência de condições clínicas para responderem aos questionamentos.

Para analisar as características individuais, foi elaborado um questionário especificamente para este estudo com dados relativos à idade, situação conjugal, condição socioeconômica, escolaridade e profissão. Para avaliar a existência da depressão nesta população, foi utilizado a Escala de Depressão Geriátrica de Yesavage em versão reduzida (GDS-15), validado internacionalmente 
inclusive para o português, recomendado pela Organização Mundial de Saúde e citada no Código Internacional de Doenças (CID-10). Os questionários foram aplicados no período de Fevereiro a Abril de 2010 e tiveram como ponto de corte os valores entre seis e dez pontos para uma possível depressão variando entre leve e moderada. Foi atribuída ao paciente com pontuação igual ou superior a onze pontos, uma condição de possível depressão grave.

Foi realizada uma análise descritiva de todas as variáveis e cruzamentos para verificação de possíveis associações das respostas. Para tanto, utilizou-se o programa SPSS-12, ficando previamente estabelecido um valor de $\mathrm{p}<0,05$ para significância estatística.

Este trabalho foi aprovado pelo Comitê de Ética em Pesquisa da Universidade Vale do Rio Verde - UNINCOR sob o número CAAE0018.0.380.000-09. Os participantes foram informados previamente sobre os objetivos da pesquisa e sobre o sigilo absoluto das informações prestadas. Aqueles que concordaram em participar assinaram previamente à aplicação do questionário um Termo de Consentimento Livre e Esclarecido em duas vias, ficando uma cópia com o participante e outra com o pesquisador.

\section{RESULTADOS E DISCUSSÃO}

Participaram deste estudo 97 idosos com idade média de 70,69 anos, sendo 72 (74,2\%) do sexo feminino e $25(25,8 \%)$ do sexo masculino. A Tabela 1 mostra as características sociodemográficas da população estudada, onde se verifica a prevalência de idosos viúvos (49,5\%), com moradia própria $(94,8 \%)$, que moram com familiares $(71,1 \%)$, que estudaram até a $4^{\mathrm{a}}$ série e não trabalham, sendo do lar.

A população estudada apresenta características semelhantes à de outros trabalhos realizados com idosos no Brasil, onde se observa uma maior frequência de mulheres, com baixa escolaridade e viúvas (FERRARI e DELACORTE, 2007; PARADELA et al., 2005; SOUZA et al., 2007). Elas vivem mais que os homens, tendem a informar mais seus problemas de saúde, apresentam menor dificuldade de admitir a presença de sintomas psicológicos e procuram mais os serviços médicos, inclusive para os problemas de menor gravidade (GUIMARÃES， 2006; GAZALLE， 2004; SOUZA et al., 2007).

Em relação à pontuação obtida na GDS, a maioria dos idosos participantes, 61 (62\%), não apresentaram características depressivas, como mostra a Tabela 2. Entretanto, 31 (32\%) obtiveram pontuação entre 6 e 10, indicando uma possível condição depressiva. Além disso, e ainda mais importante, 5 idosos $(5,2 \%)$ obtiveram pontuação superior a 11 , indicando uma possível condição de depressão grave. São encontrados na literatura, resultados que mostram taxas bem variadas para a suspeita de 
depressão em idosos (GALDINO, 2000; OLIVEIRA et al., 1993). Entretanto, é importante ressaltar que em todos os casos a sua prevalência é elevada, situação esta bastante preocupante.

Tabela 1 - Distribuição da frequência das características sócio-demográficas.

\begin{tabular}{|c|c|c|c|}
\hline & Características & $\begin{array}{l}\text { Frequência } \\
\text { (n) }\end{array}$ & $(\%)$ \\
\hline \multirow[t]{2}{*}{ Sexo } & Feminino & 72 & 74,2 \\
\hline & Masculino & 25 & 25,8 \\
\hline \multirow{3}{*}{$\begin{array}{l}\text { Situação } \\
\text { conjugal }\end{array}$} & Casado & 40 & 41,2 \\
\hline & Viúvo & 48 & 49,5 \\
\hline & Outro & 9 & 9,3 \\
\hline \multirow[t]{2}{*}{ Tipo de moradia } & Própria & 92 & 94,8 \\
\hline & Alugada/Cedida & 5 & 5,2 \\
\hline \multirow{3}{*}{$\begin{array}{l}\text { Situação } \\
\text { moradia }\end{array}$} & Sozinho & 11 & 11,3 \\
\hline & Com o companheiro & 17 & 17,5 \\
\hline & Com familiares/outros & 69 & 71,1 \\
\hline \multirow[t]{4}{*}{ Escolaridade } & Não alfabetizado & 19 & 19,6 \\
\hline & Até $4^{\mathrm{a}}$ série & 65 & 67,0 \\
\hline & Entre $5^{\mathrm{a}}$ e $8^{\mathrm{a}}$ série & 11 & 11,3 \\
\hline & Ensino Médio & 2 & 2,1 \\
\hline Profissão ou & Desempregado & 5 & 5,2 \\
\hline \multirow[t]{3}{*}{ Ocupação } & Empregado/autônomo & 5 & 5,2 \\
\hline & Aposentado & 38 & 39,2 \\
\hline & Do lar & 49 & 50,5 \\
\hline
\end{tabular}

pequeno em relação ao de mulheres, impossibilitando inferir sobre tal correlação.

$\mathrm{Na}$ literatura, são encontrados diversos trabalhos em que essa tendência também é observada. No trabalho realizado por Porcu et. al. (2002), as mulheres apresentaram maior porcentagem de sintomas depressivos graves. No estudo de Gazalle et. al. (2004), sobre a

Tabela 2 - Distribuição da frequência, segundo o gênero e a GDS-15.

\begin{tabular}{|c|c|c|c|c|c|}
\hline & & \multicolumn{3}{|c|}{ Avaliação Yesavage } & \multirow[b]{2}{*}{ Total } \\
\hline & & Normal & $\begin{array}{l}\text { Depressão } \\
\text { leve a } \\
\text { moderada }\end{array}$ & $\begin{array}{l}\text { Depressão } \\
\text { grave }\end{array}$ & \\
\hline \multirow[t]{2}{*}{ Gênero } & Feminino & $45(62,5 \%)$ & $22(30,6 \%)$ & $5(6,9 \%)$ & $72(100 \%)$ \\
\hline & Masculino & $16(64,0 \%)$ & $9(36,0 \%)$ & $0(0,0 \%)$ & 25 (100\%) \\
\hline Total & & $61(62,9 \%)$ & $31(32,0 \%)$ & $5(5,2 \%)$ & 97 (100\%) \\
\hline
\end{tabular}

Fonte: Os autores.

frequência de alguns sintomas depressivos na população idosa de Pelotas (RS), foi constatada uma média bastante elevada de sintomas depressivos, sendo que os indivíduos do sexo feminino foram os mais prevalentes (GAZALLE et al., 2004). A despeito desta tendência, são necessários estudos adicionais e específicos para que essa correlação possa ser inferida.

A alteração de certas condições como a ocorrência de doenças crônicas, viuvez e perda Além disso, chama a atenção o fato de 27 entrevistados com possível quadro depressivo serem do sexo feminino, indicando uma possível predisposição deste gênero para essa condição. Deve-se ressaltar, contudo, que o número de homens neste estudo é muito de familiares e amigos, traz a necessidade das pessoas se ajustarem a uma nova realidade, demandando uma maior capacidade de reorientação e reorganização (WALSH, 2001). A condição de viuvez, por exemplo, segundo Fernandes (FERNANDES et al., 2010), pode trazer implicações de múltiplas dimensões 
para o indivíduo, especialmente para os idosos. Nesta condição, esses indivíduos passam por um processo adaptativo que, em geral, são acompanhados por tristeza, problemas de saúde, alterações psíquicas, redução das relações interpessoais, dentre outras características. Frente a essa realidade, muitos idosos conseguem, após certo tempo, se adequar às novas circunstâncias. Porém, alguns desenvolvem formas patológicas de luto evidenciadas especialmente por doenças mentais, como a depressão. Para verificar uma possível associação entre a situação conjugal e a depressão nos indivíduos entrevistados, foi utilizado o teste Exato de Fisher não tendo sido detectada nenhuma associação estatística. Entretanto, o possível diagnóstico depressivo foi mais frequente em idosos viúvos do que em casados, como mostra a Tabela 3.

Tabela 3 - Distribuição da frequência, segundo a situação conjugal e a GDS-15.

\begin{tabular}{lccccc}
\hline \hline & & \multicolumn{4}{c}{ Avaliação Yesavage } \\
\cline { 3 - 5 } & & \multicolumn{3}{c}{ Depressão } & \multirow{2}{*}{ Total } \\
\hline Situação & Casado & $27(67,5 \%)$ & $11(27,5 \%)$ & $2(5,0 \%)$ & $40(100,0 \%)$ \\
conjugal & Viúvo & $27(56,3 \%)$ & $19(39,6 \%)$ & $2(4,2 \%)$ & $48(100,0 \%)$ \\
& Outras & $7(77,8 \%)$ & $1(11,1 \%)$ & $1(11,1 \%)$ & $9(100,0 \%)$ \\
\cline { 2 - 5 } Total & & $61(62,9 \%)$ & $31(32,0 \%)$ & $5(5,2 \%)$ & $97(100,0 \%)$ \\
\hline \hline
\end{tabular}

Fonte: Os autores.

\section{CONCLUSÕES}

Após a coleta e análise de dados, verificou-se que a frequência de suspeita de depressão entre os idosos da amostra estudada foi de 36 (37,2\%), sendo esse resultado similar a aqueles encontrados em outros estudos. O número de casos em que o sexo feminino aparece foi bem superior ao número de casos relacionados ao sexo masculino, não tendo sido verificada, contudo, nenhuma associação estatística do gênero com a condição de possível quadro depressivo. A associação da depressão com a situação conjugal também não foi encontrada.

A depressão constitui enfermidade mental frequente no idoso, comprometendo significativamente sua qualidade de vida. É uma condição que coloca em risco a vida, sobretudo daqueles que têm alguma doença crônico-degenerativa ou incapacitante, devido à existência de uma relação recíproca no diz respeito à evolução clínica do paciente. Além disso, esta é uma condição mental nem sempre diagnosticada, sendo frequentemente considerada de forma equivocada uma decorrência natural do envelhecimento e que pode levar ao agravamento de eventuais doenças orgânicas que acometem o indivíduo, aumentando a morbidade e o risco de morte. A depressão nos idosos é, por fim, um sério e crescente problema de saúde pública, trazendo prejuízos à comunidade, à vida familiar e, principalmente, ao próprio individuo. Tais fatos evidenciam a importância do desenvolvimento de estudos e projetos que incluam ações direcionadas à saúde mental das pessoas nesta fase da vida. 


\section{REFERÊNCIAS}

ALMEIDA, M. F. et al. Prevalência de doenças crônicas auto-referidas e utilização de serviços de saúde, PNAD/1998, Brasil. Cien Saúde Colet, v. 7, n. 4, p. 743-756, 2002.

AROS, M. S.; YOSHIDA, E. M. P. Estudos da depressão: Instrumentos de avaliação e gênero. Bol. psicol [on line], v. 59, n. 130, p. 61-76, 2009.

AVILA, R.; BOTTINO, C. M. D. C. Atualização sobre alterações cognitivas em idosos com síndrome depressiva. Rev Bras Psiquiatr, v. 28, n. 4, p. 316-220, 2006.

CORRÊA, A. C. D. O. Depressão e poliqueixas no idoso. J. Bras. Psiquitr., n. 46(1), p. 13-16, 1997.

DJERNES, J. K. Prevalence and predictors of depression in populations of elderly: a review. Acta Psychiatr Scand , v. 113, p. 372-387, 2006.

FERNANDES, M. G. M.; NASCIMENTO, N. F. S.; COSTA, K. N. F. M. Prevalência e determinantes de sintomas depressivos em idosos atendidos na atenção primária de saúde. Rev. RENE, v. 11, n. 1, p. 19-27, 2010.

FERRARI, J.; DALACORTE, R. R. Uso da Escala de Depressão Geriátrica de Yesavage para avaliar a prevalência de depressão em idosos hospitalizados. Sci. med., v. 17, n. 1, p. 3-8, 2007.

FLECK, M. P. A. et al. Associação entre sintomas depressivos e funcionamento social em cuidados primários à saúde. Rev Saúde Pública, v. 36, n. 4, p. 431-438, 2002.

FREITAS, E. V. Tratado de Geriatria e Gerontologia. Rio de Janeiro: Guanabara Koogan, 2002. 204-215 p.

GALDINO, J. M. S. Ansiedade, depressäo e coping em idosos. Dissertação de Mestrado. Universidade de Säo Paulo (USP), Escola de Enfermagem. [S.1.], p. 103. 2000.
GAZALLE, F. K. et al. Sintomas depressivos e fatores associados em população idosa no Sul do Brasil. Rev Saúde Pública, v. 38, n. 3, p. 365-371, 2004.

GUMARAES, J. M. N.; CALDAS, C. P. A influência da atividade física nos quadros depressivos de pessoas idosas: uma revisão sistemática. Rev Bras Epidemiol , v. 9, n. 4, p. 481-492, 2006.

IBGE - Instituto Brasileiro de Geografa e Estatística. Pesquisa Nacional por Amostras de Domicílios - PNAD 2004. Disponivel em: $<$ www.ibge.gov.br/home/estatistica/populacao /trabalhoerendimento/pnad2004/default.shtm>. Acesso em: 1 maio 2009.

IRIGARAY, T. Q.; SCHNEIDER, R. H. Prevalência de depressão em idosas participantes da Universidade para a Terceira Idade. Rev Psiquiatr, v. 29, n. 1, p. 19-27, 2007.

LEITE, V. M. M. et al. Depressão e envelhecimento: estudo nos participantes do programa Universidade Aberta à Terceira Idade. Rev Bras Saúde Mater Infantil, v. 6, n. 1, p. 31-38, 2006.

MARQUES TEIXEIRA, J. et al. Consensos Psiquiátricos para Clínicos Gerais. 1a. ed. Linda-a-Velha: Vale \& Vale Editores, 2007.

MONTORIO, I.; IZAL, M. The Geriatric Depression Scale: A Review of Its Development and Utility. International Psychogeriatrics, v. 8, n. 1, 1996.

MORAES, $H$. et al. O exercício físico no tratamento da depressão em idosos: revisão sistemática. Rev Psiquiatr RS, v. 29, n. 1, p. 70-79, 2007.

OLIVEIRA, D. A. A. P.; GOMES, L.; OLIVEIRA, R. F. Prevalência de depressão em idosos que frequentam centros de convivência. Rev. Saúde Pública, v. 40, n. 4, p. 734-736, 2006. 
OLIVEIRA, M. R. Depressão na velhice: aspectos epidemiológicos. Dissertação de Mestrado. Universidade Federal da Bahia (UFB), Escola de Enfermagem. [S.1.], p. 126. 1993.

PARADELA, E. M. P.; LOURENCO, R. A.; VERAS, R. P. Validação da escala de depressão geriátrica em um ambulatório geral. Rev de Saúde Pública, v. 39, n. 6, p. 918-923, 2005.

PAYKEL, E. S.; PRIEST, R. G. Recognition and management of depression in general practice: consensus statement. BMJ, v. 305, p. 1198-1202, 1992.

PORCU, M. et al. Estudo comparativo sobre a prevalência de sintomas depressivos em idosos hospitalizados, institucionalizados e residentes na comunidade. Acta Scientiarum, Maringá, v. 24, n. 3, p. 713-717, 2002.

RAMOS, L. R. A saúde do idoso no Brasil: Uma visão clínico epidemiológica. LivreDocência - Universidade Federal de São Paulo (UNIFESP). [S.1.]. 1997.

SHMUELY, Y. et al. Predictors of improvement in health-related quality of life among elderly patients with depression. International Psychogeriatrics, v. 13, n. 1, p. 63-73, 2001.

SILVA, M. C. O processo de envelhecimento no Brasil: desafios e perspectivas. Textos Envelhecimento, v. 8, n. 1, 2005. Disponivel em:

$<$ http://revista.unati.uerj.br/scielo.php?script=s ci_arttext\&pid=S1517-

59282005000100004\&lng=pt\&nrm=iso $>$.

Acesso em: Nov 2009.

SNOWDON, J. How high is the prevalence of depression in old age? Rev Bras Psiquiatr, v. 24, n. Supl. I, p. 42-47, 2002.

SOUSA, L.; GALANTE, H.; FIGUEIREDO, D. Qualidade de vida e bem-estar dos idosos: um estudo exploratório na população portuguesa. Rev Saúde Pública, n. 37, p. 364371, 2003.

SOUSA, R. L. et al. Validade e fidedignidade da Escala de Depressão Geriátrica na identifcação de idosos deprimidos em um hospital geral. J Bras Psiquiatr, v. 56, n. 2, p. 102-107, 2007.

STELLA, F. et al. Depressão no Idoso: Diagnóstico, tratamento e benefícios da atividade física. Motriz, Rio Claro, v. 8, n. 3, 91-98 2002., v. 8, n. 3, 91-98 2002.

TEIXEIRA, A. R. et al. Sintomatologia depressiva em deficientes auditivos adultos e idosos: importância do uso de próteses auditivas. Arq Int. Otorrinolaringol, v. 11, n. 4, p. 453-458, 2007.

VALENTINI, W. et al. Treinamento de clínicos para o diagnóstico e tratamento da depressão. Rev Saúde Pública, v. 21, n. 1, p. 522-528, 2004.

VASCONCELOS, N. P. C. Avaliação da Qualidade de Vida dos Agentes Comunitários de Saúde de Lagoa Santa/MG. Dissertação de Mestrado. Universidade Vale do Rio Verde. Programa de Pós-Graduação em Ciências da Saúde. [S.1.]. 2007.

WALSH, F. A família no Estágio Tardio da Vida. In: As mudanças no ciclo de vida familiar. Porto Alegre: Artmed, v. 1, 2001. p.512. 